\title{
The Indoor Personnel Inertial Positioning Based on Image Recognition Technology in Substation Depth
}

\author{
Min $X^{a}$, Lin PENG, Hai-Yun HAN, Ni-Ge LI, Zhan-Sheng HOU, Gang WANG, \\ Xing-Chuan BAO, He WANG and Hai YU \\ Global Energy Interconnection Research Institute, China
}

\begin{abstract}
A kind of inertial positioning technology personnel indoor substation depth based on image recognition, scene modeling using $3 \mathrm{D}$ scanning depth camera for image acquisition of fast; key position of image recognition front-end uses image recognition software algorithm, the image information is converted to dot matrix data, connect the background server to obtain accurate positioning coordinates, greatly reduce the direct image transmission to the identification of the amount of data in the deployment of additional equipment to achieve precise positioning of field personnel without the aid, effectively solve the traditional Bluetooth, millimeter wave and laser positioning need in the site layout a lot of equipment problems, eliminate safety hazards in the substation; finally, the inertial navigation algorithm, field personnel positioning using gyroscope and accelerometer do the positioning of the auxiliary, smooth, real-time and accurate, have The utility model solves the problem of poor positioning accuracy of the electric field operation, and improves the working efficiency of the field operators.
\end{abstract}

\section{Introduction}

With the development of electric power industry in our country is increasingly expanding and new equipment put into operation, operation and maintenance of power equipment maintenance information, facing complicated procedures, complicated equipment disassembly and high precision and the staff level is not a problem, the type and size of equipment in the substation is also increasing, fully enclosed substation is currently the most advanced, more profound changes in the original open substation deployment mode, all devices are placed in the substation of large and small size ranging from relatively independent closed room, operation and maintenance personnel to accurately find the target device only through experience in the field, can not accurately know its location information location and the target device, need to spend a lot of time to find the equipment, seriously affecting work the efficiency, especially in emergency situations, facing the emergency situation of substation unprepared, on-site personnel can quickly It is very important to find the location of the fault equipment quickly for the repair process, the traditional operating mode can not meet the requirements of the development of the power grid, so it is urgent to solve the problem of indoor positioning.

The substation network communication environment is more complex, often plagued by high voltage equipment electromagnetic interference and substation remote area information intensity is

\footnotetext{
${ }^{a}$ Corresponding author: xumin1@geiri.sgcc.com.cn
} 
weak and other issues, but the field of power equipment variety, modeling workload, and the model takes up more resources, all from the terminal identification is too large for the terminal pressure, therefore must by the background server real-time online image recognition. But by the communication technology, real-time live streaming video transmission to the background recognition efficiency is low, so it is necessary to study a set of front image preprocessing technology, image recognition for dot matrix information, dot matrix data transmission compression to the background recognition, greatly reduce the amount of data transmission, improve the bandwidth utilization rate.

Secondly, because of the special environment of substation and high risk equipment, determine its application in indoor positioning technology requirements as much as possible to build less auxiliary equipment, but the indoor positioning and navigation technology to traditional coordinate positioning by building auxiliary equipment, build environment is more complex and high cost, including Bluetooth positioning method, millimeter wave localization method, infrared positioning method, GPS positioning method, for the special environment of substation, cannot build various types of equipment in the field, so the traditional indoor positioning technology in substation environment not suitable.

\section{Indoor inertial positioning technology in substation depth based on image recognition}

Due to the rapid development of power equipment, including the full closed type substation, the onsite personnel demand for location information is more and more urgent, but because of the special environment of substation and high risk equipment, determine its application in indoor positioning technology requirements as much as possible to build less auxiliary equipment, this paper put forward to solve the above problems effective method for processing, using three-dimensional modeling scene scanning depth camera quickly and effectively solve the three-dimensional modeling [1] requires a lot of manual operation and operating personnel with professional knowledge of the operating problems; secondly, image acquisition of the key position of online image recognition using image recognition software algorithm, connecting the backend server to get accurate coordinate positioning, to achieve precise positioning of the auxiliary equipment on-site personnel without additional arrangement, Effectively solve the traditional Bluetooth, millimeter wave and laser positioning method [2] in the site layout a lot of equipment problems, eliminate safety hazards in the substation; finally, the inertial navigation algorithm, field personnel positioning using gyroscope and accelerometer assisted, do the personnel positioning of the smooth, real-time and accurate operation, effectively solve the positioning accuracy the electric field difference problem, improve the work efficiency of on-site personnel. Including the following steps:

Step one: using the scene modeling for 3D scanning depth camera fast, according to the modeling method of image, video and depth vision sensor data driven, 3D geometry of the two-dimensional images to restore the scene, or the use of depth vision sensor RGB-D, the three-dimensional point of continuous data frame mosaic to obtain scene cloud, realize Panorama modeling the scene in a rapid manner. The 3D depth camera using two camera while shooting photos, according to the geometrical relationship of the relationship between two images and camera image space, the actual location information of feature points, imaging principle as shown in Figure 1. If the camera projection model, image point position corresponding to determine ( $p_{1}, p_{2}$ and known), between the two outside the parameters of the camera ( $C_{1}, C_{2}$ and determine the position of known) case, only to determine the spatial position in world coordinates, the three-dimensional $P$ coordinate of the reconstruction. 


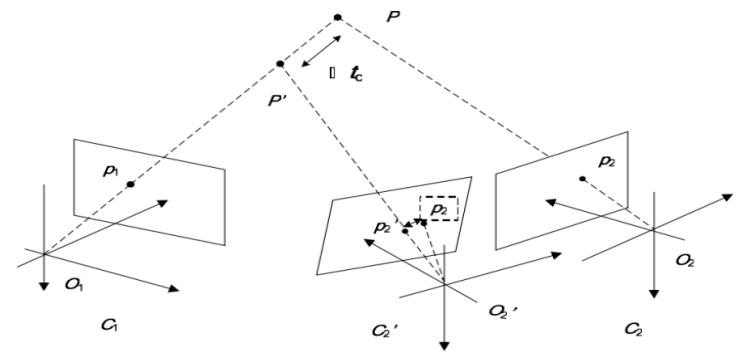

Figure 1

Step two: in the substation site selecting feature image points different as standard template matching, using feature block template matching algorithm for on-line identification of images, in front of the equipment deployment image preprocessing module [3], for each frame of the collected images were preprocessed as the sample template, the feature of template matching, the first is the transformation of the standard template and template, according to the number of feature block template is divided into a plurality of same size blocks, such as the real-time video frame has 10 feature blocks, the video frame image is divided into 64 squares average size the same, the assumption that the 10 the average distribution of feature blocks in one of the 8 boxes, the remaining 56 squares abandon feature points statistics only the remaining 8 squares, the final image is cut into dot matrix information. Through the transmission of the lattice data, the amount of data transmitted directly to a large number of video images is greatly reduced, the transmission efficiency is improved, and the bandwidth utilization rate is reduced.

Step three: terminal dot data to the backend server, the server receives the lattice data, using lattice data feature block and standard template block by block matching, then don't is small, it is not matching. As the sample $D(k)$ template and template matching in block comparison $(i, j)_{\text {results }}$ (results for 0,1 , the results do not match), $N(i, j)$ as the sample feature block template, $N_{k}^{T}(i, j)$ as a standard feature block matching template, the difference between the size of the threshold $T$, according to the threshold to identify the corresponding image model, block matching $(i, j)$ results:

$$
D(k)=\left\{\begin{array}{l}
0 N(i, j)-N_{k}^{T}(i, j) \leq T \\
1 N(i, j)-N_{k}^{T}(i, j)>T
\end{array}\right.
$$

Finally, the result is compared with the standard image feature block template

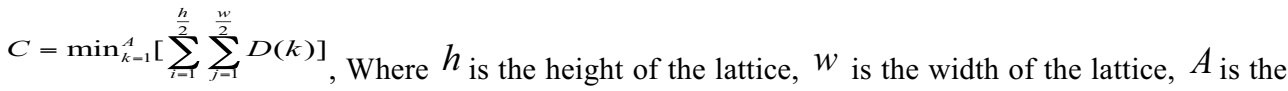
number of standard image lattice set.

Step four: through the background data to identify the current location of the image lattice number, query the database to obtain precise location coordinates, encrypted transmission to the terminal for analysis by using the SM1 cryptographic algorithm, terminal server to receive encrypted, decrypted by using the SM1 cryptographic algorithm, obtaining user coordinates where information, dynamic realtime correction of terminal guide map marker the position, to achieve precise positioning of field personnel without additional auxiliary equipment layout, the specific architecture is shown in Figure 2. 


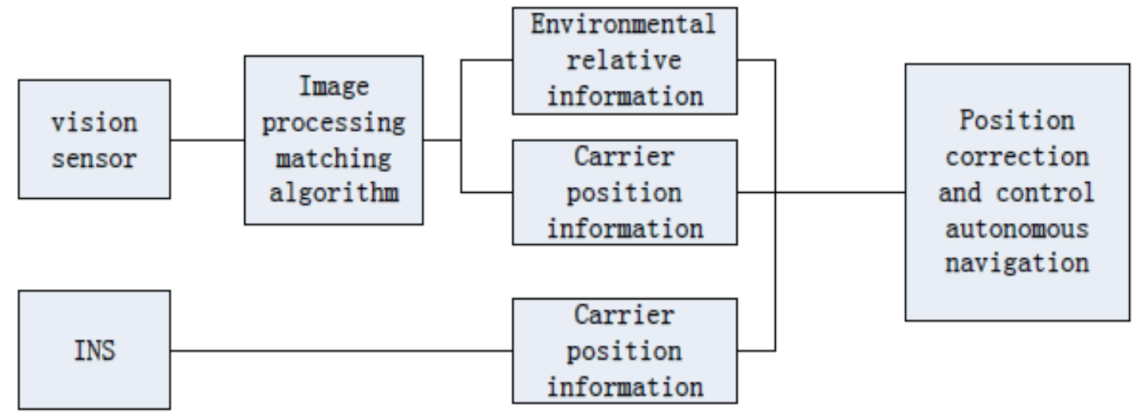

\section{Figure 2}

Step five: image recognition and localization algorithm using mathematical description of image features, in addition to the accuracy of the image error introduced by the image will show similar characteristics, so in the computer matching process, according to the characteristics of mathematical description, there will be mistakes and errors, matching, there will have an impact on the whole, the reliability of the result. The accuracy and therefore, there must be the image matching results were determined by determining the appropriate method, eliminating the false matching, to ensure the reliability of matching results; and for a certain time, can not use the image matching technology, you need to consider additional auxiliary navigation information to maintain visual navigation algorithm operation. The strapdown inertial navigation algorithm, the positioning of gyroscope and accelerometer assisted site personnel, auxiliary image recognition and positioning technology, inertial orientation modification without image marks or image recognition errors, where the smooth movement of personnel in the mobile map to mark the location of the. According to the characteristics of the substation in the power grid, a simple inertial navigation algorithm is designed to meet the needs of fast navigation.

Inertial navigation algorithm [4] is mainly divided into three parts: attitude update, speed update and location update. Its core is based on a correct attitude, so the most important impact on the accuracy of strapdown inertial navigation algorithm is the attitude update algorithm, followed by the speed update algorithm.

The attitude update algorithm is as follows:

$$
C_{b(k)}^{n}=C_{b(k-1)}^{n} C_{b(k)}^{b(k-1)} \text {, among, } C_{b(k)}^{b(k-1)} \text { When the } \mathrm{n} \text { system is used as the reference coordinate }
$$
system, $b$ is $t_{k-1}$ to $t_{k}$ transform direction cosine, $C_{b(k)}^{n}$ is $t_{k}$ attitude direction cosine, $C_{b(k-1)}^{n}$ is $t_{k-1}$ attitude direction cosine. If make $w_{n b}^{b}(t)$ the rotational angular velocity of the carrier coordinate system relative to the navigation coordinate system, $\Phi_{k_{\text {is }}} t_{m-1 \text { to }} t_{m}$ moment coordinate system $b$ equivalent rotation vector relative to navigation coordinate system, the final rotation vector is $\Phi_{k}=\int_{t_{k-1}}^{t_{k}} w_{n b}^{n}(t) d t=\int_{t_{k-1}}^{t_{k}} w_{i b}^{b}(t) \quad\left(C_{b}^{n}(t)\right)^{T} w_{i n}^{n}(t) d t$.

Speed update algorithm is as follows: 


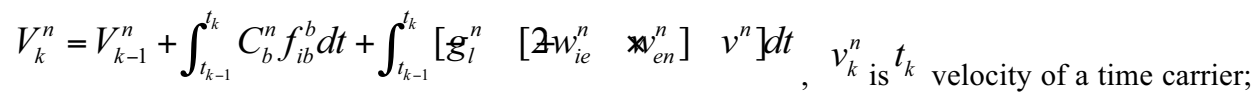

$v_{k-1 \text { is }}^{n} t_{k-1}$ velocity of a time carrier; $\int_{t_{k-1}}^{t_{k}} C_{b}^{n} f_{i b}^{b} d t$ is $t_{k-1}$ to $t_{k}$ velocity increment of time ; $\int_{t_{k-1}}^{t_{k}}\left[g_{l}^{n}-\left[2 w_{i e}^{n}+w_{e n}^{n}\right] \rtimes^{n}\right] d t{ }_{\text {is }} t_{k-1} t_{\text {to }} t_{k}$ gravity and velocity increment.

Step six: calculate the coordinates of inertial navigation based on real-time strapdown inertial navigation algorithm and terminal acceleration and gyro sensor, real-time correction of the client map marks the position, to achieve a smooth navigation in the feature image between the blank area features make navigation between images, the auxiliary field personnel for precise navigation [5].

\section{Summary}

This paper presents an indoor positioning method of substation personnel inertial depth based on image recognition, scene modeling using 3D scanning depth camera for image acquisition of fast; key position of image recognition front-end uses image recognition software algorithm, the image information is converted to dot matrix data, connect the background server to obtain accurate positioning coordinates, greatly reduce the direct the background image is transmitted to the identification data, and deployment of additional equipment to achieve precise positioning of field personnel without the aid, effectively solve the traditional Bluetooth, millimeter wave and laser positioning need in the site layout a lot of equipment problems, eliminate safety hazards in the substation; finally, the inertial navigation algorithm, field personnel positioning by using gyroscope and the acceleration sensor, do smooth, real-time positioning And accurately and effectively solve the problem of poor positioning accuracy of electric field operation, and improve the working efficiency of field workers.

\section{References}

1. Infinite reality avatars, eternal life, new worlds, and the dawn of the virtual revolution. J. Blascovich,J. B. Bailenson. . 2011

2. Power,madness and immortality:the future of virtual reality. Mychilo Stephenson Cline. . 2005

3. Exodus to the Virtual World:How Online Fun is Changing Reality. Castronova,Edward. . 2007

4. An introduction to the finite element method. J. N. Reddy. . 1993

5. The CMS experiment at the CERN LHC. J.?Instrum. 2008 